\title{
Application of Maple on the Differential Problem
}

\author{
Chii-Huei Yu \\ Department of Management and Information, Nan Jeon University of Science and Technology, Tainan City, 73746, Taiwan \\ *Corresponding Author: chiihuei@mail.nju.edu.tw
}

Copyright $@ 2014$ Horizon Research Publishing All rights reserved.

\begin{abstract}
This paper uses the mathematical software Maple for the auxiliary tool to study the differential problem of two types of functions. We can obtain the infinite series forms of any order derivatives of these two types of functions by using Leibniz differential rule, differentiation term by term, and integration term by term. And hence greatly reduce the difficulty of calculating the higher order derivative values of these functions. On the other hand, we propose two examples to do calculation practically. The research methods adopted in this study involved finding solutions through manual calculations and verifying these solutions by using Maple. This type of research method not only allows the discovery of calculation errors, but also helps modify the original directions of thinking from manual and Maple calculations. For this reason, Maple provides insights and guidance regarding problem-solving methods.
\end{abstract}

Keywords Derivatives, Infinite Series Forms, Leibniz Differential Rule, Differentiation Term By Term, Integration Term By Term, Maple

\section{Introduction}

As information technology advances, whether computers can become comparable with human brains to perform abstract tasks, such as abstract art similar to the paintings of Picasso and musical compositions similar to those of Mozart, is a natural question. Currently, this appears unattainable. In addition, whether computers can solve abstract and difficult mathematical problems and develop abstract mathematical theories such as those of mathematicians also appears unfeasible. Nevertheless, in seeking for alternatives, we can study what assistance mathematical software can provide. This study introduces how to conduct mathematical research using the mathematical software Maple. The main reasons of using Maple in this study are its simple instructions and ease of use, which enable beginners to learn the operating techniques in a short period. By employing the powerful computing capabilities of Maple, difficult problems can be easily solved. Even when Maple cannot determine the solution, problem-solving hints can be identified and inferred from the approximate values calculated and solutions to similar problems, as determined by Maple. For this reason, Maple can provide insights into scientific research. Inquiring through an online support system provided by Maple or browsing the Maple website (www.maplesoft.com) can facilitate further understanding of Maple and might provide unexpected insights. For the instructions and operations of Maple, [1-7] can be adopted as references.

In calculus and engineering mathematics curricula, evaluating the $n$-th order derivative value $f^{(n)}(c)$ of a function $f(x)$ at $x=c$, in general, needs to go through two procedures: firstly determining the $n$-th order derivative $f^{(n)}(x)$ of $f(x)$, and then taking $x=c$ into $f^{(n)}(x)$. These two procedures will make us face with increasingly complex calculations when calculating higher order derivative values of this function ( i.e. $n$ is large), and hence to obtain the answers by manual calculations is not easy. In this paper, we mainly study the differential problem of the following two types of functions

$$
\begin{aligned}
& f(x)=e^{a x} x^{b} \tan ^{-1} x^{c} \\
& g(x)=e^{a x} x^{b} \cot ^{-1} x^{c}
\end{aligned}
$$

Where $a, b, c$ are real numbers. We can obtain the infinite series forms of any order derivatives of these two types of functions by using Leibniz differential rule, differentiation term by term, and integration term by term ; these are the major results of this study (i.e., Theorems 1 and 2), and hence greatly reduce the difficulty of calculating their higher order derivative values. For the study of related differential problems can refer to [8-21]. In addition, we provide two examples to do calculation practically. The research methods adopted in this study involved finding solutions through manual calculations and verifying these solutions by using Maple. This type of research method not only allows the discovery of calculation errors, but also helps modify the original directions of thinking from manual and Maple calculations. Therefore, Maple provides insights and guidance regarding problem-solving methods.

\section{Main Results}


Firstly, we introduce a notation and a formula used in this paper.

\subsection{Notation}

Suppose $r$ is any real number, $m$ is any positive integer. Define $(r)_{m}=r(r-1) \cdots(r-m+1)$, and $(r)_{0}=1$.

\subsection{Geometric series}

If $u$ is any real number, $|u|<1$. Then

$$
\frac{1}{1+u}=\sum_{m=0}^{\infty}(-1)^{m} u^{m}
$$

Next, we introduce three important theorems used in this study.

\subsection{Differentiation term by term ([22, p230])}

If, for all non-negative integer $k$, the functions $g_{k}:(a, b) \rightarrow R$ satisfy the following three conditions : (i) there exists a point $x_{0} \in(a, b)$ such that $\sum_{k=0}^{\infty} g_{k}\left(x_{0}\right)$ is convergent, (ii) all functions $g_{k}(x)$ are differentiable on open interval $(a, b)$, (iii) $\sum_{k=0}^{\infty} \frac{d}{d x} g_{k}(x)$ is uniformly convergent on $(a, b)$. Then $\sum_{k=0}^{\infty} g_{k}(x)$ is uniformly convergent and differentiable on $(a, b)$. Moreover, its derivative $\frac{d}{d x} \sum_{k=0}^{\infty} g_{k}(x)=\sum_{k=0}^{\infty} \frac{d}{d x} g_{k}(x)$.

\subsection{Integration term by term ([22, p269])}

Suppose $\left\{g_{n}\right\}_{n=0}^{\infty}$ is a sequence of Lebesgue integrable functions defined on an inteval $I$. If $\sum_{n=0}^{\infty} \int_{I}\left|g_{n}\right|$ is convergent, then $\int_{I} \sum_{n=0}^{\infty} g_{n}=\sum_{n=0}^{\infty} \int_{I} g_{n}$.

\subsection{Leibniz differential rule ([22, p121])}

Suppose $n$ is an non-negative integer, $f(x)$ and $g(x)$ are $n$-times differentiable functions. Then the $n$-th order derivative of the product function $f(x) \cdot g(x)$,

$$
(f \cdot g)^{(n)}=\sum_{k=0}^{n}\left(\begin{array}{l}
n \\
k
\end{array}\right) f^{(n-k)} g^{(k)},
$$

where $\left(\begin{array}{l}n \\ k\end{array}\right)=\frac{n !}{k !(n-k) !}$.

Before deriving the first major results in this study, we need a lemma.

\subsection{Lemma 1}

Suppose $y$ is any real number. Then the inverse tangent function

$$
\begin{gathered}
\tan ^{-1} y \\
=\sum_{m=0}^{\infty} \frac{(-1)^{m}}{2 m+1} y^{2 m+1} \quad \text { if }|y| \leq 1 \\
=-\sum_{m=0}^{\infty} \frac{(-1)^{m}}{2 m+1} y^{-2 m-1}+\frac{\pi}{2} \quad \text { if } y \geq 1 \\
=-\sum_{m=0}^{\infty} \frac{(-1)^{m}}{2 m+1} y^{-2 m-1}-\frac{\pi}{2} \quad \text { if } y \leq-1
\end{gathered}
$$

\subsubsection{Proof}

Because the derivative of $\tan ^{-1} y$,

$$
\frac{d}{d y} \tan ^{-1} y=\frac{1}{1+y^{2}}
$$

If $|y|<1$, then

$$
\begin{gathered}
\frac{1}{1+y^{2}} \\
=\sum_{m=0}^{\infty}(-1)^{m} y^{2 m} \quad \text { (By geometric series) }
\end{gathered}
$$

Using integration term by term, we obtain

$$
\tan ^{-1} y=\sum_{m=0}^{\infty} \frac{(-1)^{m}}{2 m+1} y^{2 m+1} .
$$

On the other hand, when $y= \pm 1$, (3) holds. Thus,

$$
\tan ^{-1} y=\sum_{m=0}^{\infty} \frac{(-1)^{m}}{2 m+1} y^{2 m+1} \text { for }|y| \leq 1 .
$$

If $y>1$, because

$$
\begin{gathered}
\frac{1}{1+y^{2}} \\
=\frac{1}{y^{2}} \cdot \frac{1}{1+1 / y^{2}}
\end{gathered}
$$$$
=\frac{1}{y^{2}} \cdot \sum_{m=0}^{\infty}(-1)^{m}\left(1 / y^{2}\right)^{m}
$$

(By geometric series) 


$$
=\sum_{m=0}^{\infty}(-1)^{m} y^{-2 m-2}
$$

Therefore, by integration term by term, we have

$$
\tan ^{-1} y=-\sum_{m=0}^{\infty} \frac{(-1)^{m}}{2 m+1} y^{-2 m-1}+C
$$

Where $C$ is a constant.

Taking $y=1$, we obtain $C=\pi / 2$. Therefore,

$$
\tan ^{-1} y=-\sum_{m=0}^{\infty} \frac{(-1)^{m}}{2 m+1} y^{-2 m-1}+\frac{\pi}{2} \quad \text { for } y \geq 1 .
$$

The case of $y \leq-1$, taking $y=-1$ into (8), we obtain $C=-\pi / 2$. Thus,

$$
\tan ^{-1} y=-\sum_{m=0}^{\infty} \frac{(-1)^{m}}{2 m+1} y^{-2 m-1}-\frac{\pi}{2} \quad \text { for } y \leq-1
$$

The following is the first major result in this study, we determine the infinite series forms of any order derivatives of the function (1).

\subsection{Theorem 1}

Let $a, b, c$ be real numbers. Suppose the domain of the function

$$
f(x)=e^{a x} x^{b} \tan ^{-1} x^{c}
$$

is $\left\{x \in R \mid x^{b}, x^{c}\right.$ exist $\left.,\left|x^{c}\right| \neq 1, x \neq 0\right\}$.

Case (1): If $\left|x^{c}\right|<1$, and $x \neq 0$. Then the $n_{\text {-th order }}$ derivative of $f(x)$,

$$
=e^{a x} \sum_{k=0}^{n}\left(\begin{array}{l}
n \\
k
\end{array}\right) a^{n-k} \sum_{m=0}^{\infty} \frac{(-1)^{m}(2 c m+c+b)}{2 m+1} x^{2 c m+c+b-k}
$$

Case (2): If $x^{c}>1$, then

$$
\begin{gathered}
f^{(n)}(x) \\
=\frac{\pi}{2} e^{a x} \sum_{k=0}^{n}\left(\begin{array}{l}
n \\
k
\end{array}\right) a^{n-k}(b)_{k} x^{b-k} \\
-e^{a x} \sum_{k=0}^{n}\left(\begin{array}{l}
n \\
k
\end{array}\right) a^{n-k} \sum_{m=0}^{\infty} \frac{(-1)^{m}(-2 c m-c+b)_{k}}{2 m+1} x^{-2 c m-c+b-k}
\end{gathered}
$$

Case (3): If $x^{c}<-1$, then

$$
\begin{gathered}
f^{(n)}(x) \\
=-\frac{\pi}{2} e^{a x} \sum_{k=0}^{n}\left(\begin{array}{l}
n \\
k
\end{array}\right) a^{n-k}(b)_{k} x^{b-k}
\end{gathered}
$$

$$
-e^{a x} \sum_{k=0}^{n}\left(\begin{array}{l}
n \\
k
\end{array}\right) a^{n-k} \sum_{m=0}^{\infty} \frac{(-1)^{m}(-2 c m-c+b)_{k}}{2 m+1} x^{-2 c m-c+b-k}
$$

\subsubsection{Proof}

Case (1): If $\left|x^{c}\right|<1$, then by (3) of Lemma 1, we have

$$
\begin{gathered}
f(x)=e^{a x} x^{b} \tan ^{-1} x^{c} \\
=e^{a x} x^{b} \cdot \sum_{m=0}^{\infty} \frac{(-1)^{m}}{2 m+1}\left(x^{c}\right)^{2 m+1} \\
=e^{a x} \cdot \sum_{m=0}^{\infty} \frac{(-1)^{m}}{2 m+1} x^{2 c m+c+b}
\end{gathered}
$$

Differentiating $n$-times with respect to $x$ on both sides of (12), we obtain

$$
\begin{gathered}
f^{(n)}(x) \\
=\sum_{k=0}^{n}\left(\begin{array}{l}
n \\
k
\end{array}\right)\left(e^{a x}\right)^{(n-k)} \cdot\left(\sum_{m=0}^{\infty} \frac{(-1)^{m}}{2 m+1} x^{2 c m+c+b}\right)^{(k)}
\end{gathered}
$$

( By Leibniz differential rule)

$$
=e^{a x} \sum_{k=0}^{n}\left(\begin{array}{l}
n \\
k
\end{array}\right) a^{n-k} \sum_{m=0}^{\infty} \frac{(-1)^{m}(2 c m+c+b)_{k}}{2 m+1} x^{2 c m+c+b-k}
$$

(By differentiation term by term)

Case (2): If $x^{c}>1$, then by (4) of Lemma 1, we have

$$
\begin{gathered}
f(x)=e^{a x} x^{b} \tan ^{-1} x^{c} \\
=e^{a x} x^{b} \cdot\left(\frac{\pi}{2}-\sum_{m=0}^{\infty} \frac{(-1)^{m}}{2 m+1}\left(x^{c}\right)^{-2 m-1}\right) \\
=e^{a x}\left(\frac{\pi}{2} x^{b}-\sum_{m=0}^{\infty} \frac{(-1)^{m}}{2 m+1} x^{-2 c m-c+b}\right)
\end{gathered}
$$

Differentiating $n$-times with respect to $x$ on both sides of (13), we obtain

$$
\begin{gathered}
f^{(n)}(x) \\
=\sum_{k=0}^{n}\left(\begin{array}{l}
n \\
k
\end{array}\right)\left(e^{a x}\right)^{(n-k)} \cdot\left(\frac{\pi}{2} x^{b}-\sum_{m=0}^{\infty} \frac{(-1)^{m}}{2 m+1} x^{-2 c m-c+b}\right)^{(k)}
\end{gathered}
$$

( By Leibniz differential rule)

$$
=\frac{\pi}{2} e^{a x} \sum_{k=0}^{n}\left(\begin{array}{l}
n \\
k
\end{array}\right) a^{n-k}(b)_{k} x^{b-k}
$$

$-e^{a x} \sum_{k=0}^{n}\left(\begin{array}{l}n \\ k\end{array}\right) a^{n-k} \sum_{m=0}^{\infty} \frac{(-1)^{m}(-2 c m-c+b)_{k}}{2 m+1} x^{-2 c m-c+b-k}$

(By differentiation term by term)

Case (3): If $x^{c}<-1$, by (5) of Lemma 1, we obtain 
$f(x) \quad$ Let the assumptions be the same as Theorem 1. Suppose

$$
\begin{array}{r}
=e^{a x} x^{b} \tan ^{-1} x^{c} \\
=e^{a x} x^{b} \cdot\left(-\frac{\pi}{2}-\sum_{m=0}^{\infty} \frac{(-1)^{m}}{2 m+1}\left(x^{c}\right)^{-2 m-1}\right) \\
=e^{a x}\left(-\frac{\pi}{2} x^{b}-\sum_{m=0}^{\infty} \frac{(-1)^{m}}{2 m+1} x^{-2 c m-c+b}\right)
\end{array}
$$

Differentiating $n$-times with respect to $x$ on both sides of (14), we have

$$
=\sum_{k=0}^{n}\left(\begin{array}{l}
n \\
k
\end{array}\right)\left(e^{a x}\right)^{(n-k)} \cdot\left(-\frac{\pi}{2} x^{b}-\sum_{m=0}^{\infty} \frac{(-1)^{m}}{2 m+1} x^{-2 c m-c+b}\right)^{(k)}
$$

( By Leibniz differential rule)

$$
\begin{array}{r}
=-\frac{\pi}{2} e^{a x} \sum_{k=0}^{n}\left(\begin{array}{l}
n \\
k
\end{array}\right) a^{n-k}(b)_{k} x^{b-k} \\
-e^{a x} \sum_{k=0}^{n}\left(\begin{array}{l}
n \\
k
\end{array}\right) a^{n-k} \sum_{m=0}^{\infty} \frac{(-1)^{m}(-2 c m-c+b)_{k}}{2 m+1} x^{-2 c m-c+b-k}
\end{array}
$$

(By differentiation term by term)

Also, to obtain the second major results in this study, we need a lemma.

\subsection{Lemma 2}

Suppose $y$ is any real number. Then the inverse cotangent function

$$
\begin{array}{rl}
\cot ^{-1} & y \\
= & \frac{\pi}{2}-\sum_{m=0}^{\infty} \frac{(-1)^{m}}{2 m+1} y^{2 m+1} \quad \text { if }|y| \leq 1 \\
= & \sum_{m=0}^{\infty} \frac{(-1)^{m}}{2 m+1} y^{-2 m-1} \quad \text { if } y \geq 1 \\
=\pi+\sum_{m=0}^{\infty} \frac{(-1)^{m}}{2 m+1} y^{-2 m-1} & \text { if } y \leq-1
\end{array}
$$

\subsubsection{Proof}

Because $\cot ^{-1} y=\frac{\pi}{2}-\tan ^{-1} y$, then by Lemma 1 , we immediately obtain this result.

The following is the second major result in this paper, we obtain the infinite series forms of any order derivatives of the function (2).

\subsection{Theorem 2}




$$
\begin{gathered}
=\frac{\pi}{2} e^{a x} \sum_{k=0}^{n}\left(\begin{array}{l}
n \\
k
\end{array}\right) a^{n-k}(b)_{k} x^{b-k} \\
-e^{a x} \sum_{k=0}^{n}\left(\begin{array}{l}
n \\
k
\end{array}\right) a^{n-k} \sum_{m=0}^{\infty} \frac{(-1)^{m}(2 c m+c+b)_{k}}{2 m+1} x^{2 c m+c+b-k}
\end{gathered}
$$

(By differentiation term by term)

Case (2): If $x^{c}>1$, then by (16) of Lemma 2, we have

$$
\begin{gathered}
g(x) \\
=e^{a x} x^{b} \cot ^{-1} x^{c} \\
=e^{a x} x^{b} \cdot \sum_{m=0}^{\infty} \frac{(-1)^{m}}{2 m+1}\left(x^{c}\right)^{-2 m-1} \\
=e^{a x} \cdot \sum_{m=0}^{\infty} \frac{(-1)^{m}}{2 m+1} x^{-2 c m-c+b}
\end{gathered}
$$

Differentiating $n$-times with respect to $x$ on both sides of (22), we obtain

$$
\begin{gathered}
g^{(n)}(x) \\
=\sum_{k=0}^{n}\left(\begin{array}{l}
n \\
k
\end{array}\right)\left(e^{a x}\right)^{(n-k)} \cdot\left(\sum_{m=0}^{\infty} \frac{(-1)^{m}}{2 m+1} x^{-2 c m-c+b}\right)^{(k)}
\end{gathered}
$$

( By Leibniz differential rule)

$$
=e^{a x} \sum_{k=0}^{n}\left(\begin{array}{l}
n \\
k
\end{array}\right) a^{n-k} \sum_{m=0}^{\infty} \frac{(-1)^{m}(-2 c m-c+b)_{k}}{2 m+1} x^{-2 c m-c+b-k}
$$

( By differentiation term by term)

Case (3): If $x^{c}<-1$, then by (17) of Lemma 2, we obtain

$$
\begin{gathered}
g(x) \\
=e^{a x} x^{b} \cot ^{-1} x^{c} \\
=e^{a x} x^{b} \cdot\left(\pi+\sum_{m=0}^{\infty} \frac{(-1)^{m}}{2 m+1}\left(x^{c}\right)^{-2 m-1}\right) \\
=e^{a x}\left(\pi x^{b}+\sum_{m=0}^{\infty} \frac{(-1)^{m}}{2 m+1} x^{-2 c m-c+b}\right)
\end{gathered}
$$

Differentiating $n$-times with respect to $x$ on both sides of (23), we have

$$
\begin{gathered}
g^{(n)}(x) \\
=\sum_{k=0}^{n}\left(\begin{array}{l}
n \\
k
\end{array}\right)\left(e^{a x}\right)^{(n-k)} \cdot\left(\pi x^{b}+\sum_{m=0}^{\infty} \frac{(-1)^{m}}{2 m+1} x^{-2 c m-c+b}\right)^{(k)}
\end{gathered}
$$

( By Leibniz differential rule)

$$
=\pi e^{a x} \sum_{k=0}^{n}\left(\begin{array}{l}
n \\
k
\end{array}\right) a^{n-k}(b)_{k} x^{b-k}
$$

$+e^{a x} \sum_{k=0}^{n}\left(\begin{array}{l}n \\ k\end{array}\right) a^{n-k} \sum_{m=0}^{\infty} \frac{(-1)^{m}(-2 c m-c+b)_{k}}{2 m+1} x^{-2 c m-c+b-k}$

(By differentiation term by term)

\section{Examples}

In the following, for the differential problem of the two types of functions in this study, we provide two examples and use Theorems 1,2 to determine the infinite series forms of any order derivatives and some higher order derivative values of these functions. On the other hand, we employ Maple to calculate the approximations of these higher order derivative values and their solutions for verifying our answers.

\subsection{Example 1}

Suppose the domain of the function

$$
f(x)=e^{6 x} x^{8 / 5} \tan ^{-1} x^{3}
$$

is $\{x \in R \mid x \neq 0,1,-1\}$ (the case of $a=6, b=\frac{8}{5}, c=3$ in Theorem 1).

3.1.1. If $|x|<1$ and $x \neq 0$. By Case (1) of Theorem 1, we obtain any $n$-th order derivative of $f(x)$,

$$
\begin{gathered}
f^{(n)}(x) \\
=e^{6 x} \sum_{k=0}^{n}\left(\begin{array}{l}
n \\
k
\end{array}\right) 6^{n-k} \sum_{m=0}^{\infty} \frac{(-1)^{m}\left(6 m+\frac{23}{5}\right)_{k}}{2 m+1} x^{6 m+\frac{23}{5}-k}
\end{gathered}
$$

Thus, we can evaluate the 7-th order derivative value of $f(x)$ at $x=1 / 2$,

$$
\begin{gathered}
f^{(7)}\left(\frac{1}{2}\right) \\
=e^{3} \sum_{k=0}^{7}\left(\begin{array}{l}
7 \\
k
\end{array}\right) 6^{7-k} \sum_{m=0}^{\infty} \frac{(-1)^{m}\left(6 m+\frac{23}{5}\right)}{2 m+1}\left(\frac{1}{2}\right)^{6 m+\frac{23}{5}-k}
\end{gathered}
$$

We use Maple to verify the correctness of (26) as follows: $>\mathrm{f}:=\mathrm{x}->\exp \left(6^{*} \mathrm{x}\right)^{*}\left(\mathrm{x}^{\wedge} 8\right)^{\wedge}(1 / 5)^{*} \arctan \left(\mathrm{x}^{\wedge} 3\right)$;

$$
f:=x \rightarrow \mathrm{e}^{6 x}\left(x^{8}\right)^{1 / 5} \arctan \left(x^{3}\right)
$$

>evalf((D@@7)(f)(1/2),18);

$$
2.10967839453902383 \cdot 10^{7}
$$

$>\operatorname{evalf}\left(\exp (3)^{*} \operatorname{sum}\left(\operatorname{sum}\left(7 ! /(\mathrm{k} ! *(7-\mathrm{k}) !)^{*} 6^{\wedge}(7-\mathrm{k}) *(-1)^{\wedge} \mathrm{m}^{*}\right.\right.\right.$ product $(6 * \mathrm{~m}+23 / 5-\mathrm{j}, \mathrm{j}=0 . .(\mathrm{k}-1)) /(2 * \mathrm{~m}+1) *(1 / 2)^{\wedge}(6 * \mathrm{~m}+23$

(5-k), $\mathrm{m}=0$. infinity), $\mathrm{k}=0 . .7), 18$ ); 


\section{$2.10967839453902383 \cdot 10^{7}$}

3.1.2. If $x>1$. By Case (2) of Theorem 1, we obtain any $n$-th order derivative of $f(x)$,

$$
\begin{gathered}
f^{(n)}(x) \\
=\frac{\pi}{2} e^{6 x} \sum_{k=0}^{n}\left(\begin{array}{l}
n \\
k
\end{array}\right) 6^{n-k}\left(\frac{8}{5}\right)_{k} x^{\frac{8}{5}-k} \\
-e^{6 x} \sum_{k=0}^{n}\left(\begin{array}{l}
n \\
k
\end{array}\right) 6^{n-k} \sum_{m=0}^{\infty} \frac{(-1)^{m}\left(-6 m-\frac{7}{5}\right)_{k}}{2 m+1} x^{-6 m-\frac{7}{5}-k}
\end{gathered}
$$

Hence, we can determine the 9-th order derivative value of $f(x)$ at $x=11 / 3$,

$$
\begin{gathered}
f^{(9)}\left(\frac{11}{3}\right) \\
=\frac{\pi}{2} e^{22} \sum_{k=0}^{9}\left(\begin{array}{l}
9 \\
k
\end{array}\right) 6^{9-k}\left(\frac{8}{5}\right)_{k}\left(\frac{11}{3}\right)^{\frac{8}{5}-k} \\
-e^{22} \sum_{k=0}^{9}\left(\begin{array}{l}
9 \\
k
\end{array}\right) 6^{9-k} \sum_{m=0}^{\infty} \frac{(-1)^{m}\left(-6 m-\frac{7}{5}\right)_{k}}{2 m+1}\left(\frac{11}{3}\right)^{-6 m-\frac{7}{5}-k}
\end{gathered}
$$

Also, we use Maple to verify the correctness of (28). >evalf((D@@9)(f)(11/3),22);

$$
7.782870214427535515513 \cdot 10^{17}
$$

$>$ evalf $\left(\mathrm{Pi} / 2 * \exp (22) * \operatorname{sum}\left(9 ! /(\mathrm{k} ! *(9-\mathrm{k}) !)^{*} 6^{\wedge}(9-\mathrm{k}) * \operatorname{product}(8\right.\right.$ $\left.15-\mathrm{j}, \mathrm{j}=0 . .(\mathrm{k}-1))^{*}(11 / 3)^{\wedge}(8 / 5-\mathrm{k}), \mathrm{k}=0 . .9\right)-\exp (22)^{*} \operatorname{sum}(\operatorname{sum}(9$ ! $/(\mathrm{k} ! *(9-\mathrm{k}) !) * 6^{\wedge}(9-\mathrm{k})^{*}(-1)^{\wedge} \mathrm{m} * \operatorname{product}\left(-6^{*} \mathrm{~m}-7 / 5-\mathrm{p}, \mathrm{p}=0 . .(\mathrm{k}-1)\right.$ )$/(2 * \mathrm{~m}+1) *(11 / 3)^{\wedge}(-6 * \mathrm{~m}-7 / 5-\mathrm{k}), \mathrm{m}=0$..infinity $\left.\left.), \mathrm{k}=0 . .9\right), 22\right)$;

$$
7.782870214427535515505 \cdot 10^{17}
$$

3.1.3. If $x<-1$. By Case (3) of Theorem 1, we obtain any $n$-th order derivative of $f(x)$,

$$
\begin{gathered}
f^{(n)}(x) \\
=-\frac{\pi}{2} e^{6 x} \sum_{k=0}^{n}\left(\begin{array}{l}
n \\
k
\end{array}\right) 6^{n-k}\left(\frac{8}{5}\right)_{k} x^{\frac{8}{5}-k} \\
-e^{6 x} \sum_{k=0}^{n}\left(\begin{array}{l}
n \\
k
\end{array}\right) 6^{n-k} \sum_{m=0}^{\infty} \frac{(-1)^{m}\left(-6 m-\frac{7}{5}\right)_{k}}{2 m+1} x^{-6 m-\frac{7}{5}-k}
\end{gathered}
$$

Thus, the 10-th order derivative value of $f(x)$ at $x=-2$,

$$
f^{(10)}(-2)
$$

$$
\begin{gathered}
=-\frac{\pi}{2} e^{-12} \sum_{k=0}^{10}\left(\begin{array}{c}
10 \\
k
\end{array}\right) 6^{10-k}\left(\frac{8}{5}\right)_{k}(-2)^{\frac{8}{5}-k} \\
-e^{-12} \sum_{k=0}^{10}\left(\begin{array}{c}
10 \\
k
\end{array}\right) 6^{10-k} \sum_{m=0}^{\infty} \frac{(-1)^{m}\left(-6 m-\frac{7}{5}\right)_{k}}{2 m+1}(-2)^{-6 m-\frac{7}{5}-k}(30)
\end{gathered}
$$

Using Maple to verify the correctness of (30) as follows: >evalf((D@@10)(f)(-2),14);

\subsection{8}

$>\operatorname{evalf}\left(-\mathrm{Pi} / 2 * \exp (-12) * \operatorname{sum}\left(10 ! /(\mathrm{k} ! *(10-\mathrm{k}) !) * 6^{\wedge}(10-\mathrm{k}) *\right.\right.$ product $\left.(8 / 5-\mathrm{j}, \mathrm{j}=0 . .(\mathrm{k}-1))^{*} 256^{\wedge}(1 / 5)^{*}(-1 / 2)^{\wedge} \mathrm{k}, \mathrm{k}=0 . .10\right)-\exp (-$ $12)^{*} \operatorname{sum}\left(\operatorname{sum}\left(10 ! /(\mathrm{k} ! *(10-\mathrm{k}) !) * 6^{\wedge}(10-\mathrm{k}) *(-1)^{\wedge} \mathrm{m}^{*} \operatorname{product}(-\right.\right.$ $6 * \mathrm{~m}-7 / 5-\mathrm{p}, \mathrm{p}=0 . .(\mathrm{k}-1)) /(2 * \mathrm{~m}+1)^{*}(1 / 64)^{\wedge} \mathrm{m}^{*}\left(-(1 / 128)^{\wedge}(1 / 5)\right)$ $*(-1 / 2)^{\wedge} \mathrm{k}, \mathrm{m}=0$. .infinity $\left.\left.), \mathrm{k}=0 . .10\right), 14\right)$;

\subsection{6}

\subsection{Example 2}

Let the domain of the function

$$
g(x)=e^{4 x} x^{10 / 3} \cot ^{-1} x^{5}
$$

be $\{x \in R \mid x \neq 0,1,-1\}$ (the case of $a=4, b=\frac{10}{3}, c=5$ in Theorem 2).

3.2.1. If $|x|<1$ and $x \neq 0$. By Case (1) of Theorem 2, we obtain any $n$-th order derivative of $g(x)$,

$$
\begin{gathered}
g^{(n)}(x) \\
=\frac{\pi}{2} e^{4 x} \sum_{k=0}^{n}\left(\begin{array}{l}
n \\
k
\end{array}\right) 4^{n-k}\left(\frac{10}{3}\right)_{k} x^{\frac{10}{3}-k} \\
-e^{4 x} \sum_{k=0}^{n}\left(\begin{array}{l}
n \\
k
\end{array}\right) 4^{n-k} \sum_{m=0}^{\infty} \frac{(-1)^{m}\left(10 m+\frac{25}{3}\right)_{k}}{2 m+1} x^{10 m+\frac{25}{3}-k}
\end{gathered}
$$

Therefore, the 7-th order derivative value of $g(x)$ at $x=2 / 3$,

$$
\begin{gathered}
g^{(7)}\left(\frac{2}{3}\right) \\
=\frac{\pi}{2} e^{\frac{8}{3}} \sum_{k=0}^{7}\left(\begin{array}{l}
7 \\
k
\end{array}\right) 4^{7-k}\left(\frac{10}{3}\right)_{k}\left(\frac{2}{3}\right)^{\frac{10}{3}-k} \\
-e^{\frac{8}{3}} \sum_{k=0}^{7}\left(\begin{array}{l}
7 \\
k
\end{array}\right) 4^{7-k} \sum_{m=0}^{\infty} \frac{(-1)^{m}\left(10 m+\frac{25}{3}\right)_{k}}{2 m+1}\left(\frac{2}{3}\right)^{10 m+\frac{25}{3}-k}
\end{gathered}
$$

We employ Maple to verify the correctness of (33) as follows:

$>\mathrm{g}:=\mathrm{x}->\exp \left(4^{*} \mathrm{x}\right)^{*}\left(\mathrm{x}^{\wedge} 10\right)^{\wedge}(1 / 3)^{*} \operatorname{arccot}\left(\mathrm{x}^{\wedge} 5\right)$; 


$$
g:=x \rightarrow \mathrm{e}^{4 x}\left(x^{10}\right)^{1 / 3} \operatorname{arccot}\left(x^{5}\right)
$$

>evalf((D@@)(g)(2/3),18);

$$
-2.49475867442111254 \cdot 10^{6}
$$

$>$ evalf $(\mathrm{Pi} / 2 * \exp (8 / 3) * \operatorname{sum}(7 ! /(\mathrm{k} ! *(7-\mathrm{k}) !) * 4 \wedge(7-\mathrm{k}) * \operatorname{product}($ $\left.10 / 3-\mathrm{j}, \mathrm{j}=0 . .(\mathrm{k}-1))^{*}(2 / 3)^{\wedge}(10 / 3-\mathrm{k}), \mathrm{k}=0 . .7\right)-\exp (8 / 3)^{*} \operatorname{sum}(\operatorname{sum}$ $\left(7 ! /(\mathrm{k} ! *(7-\mathrm{k}) !) * 4^{\wedge}(7-\mathrm{k})^{*}(-1)^{\wedge} \mathrm{m}^{*} \operatorname{product}\left(10^{*} \mathrm{~m}+25 / 3-\mathrm{p}, \mathrm{p}=0 .\right.\right.$. $(\mathrm{k}-1)) /(2 * \mathrm{~m}+1) *(2 / 3)^{\wedge}(10 * \mathrm{~m}+25 / 3-\mathrm{k}), \mathrm{m}=0$..infinity), $\left.\mathrm{k}=0 . .7\right)$ ,18);

$$
-2.49475867442111253 \cdot 10^{6}
$$

3.2.2. If $x>1$. By Case (2) of Theorem 2, we can determine any $n$-th order derivative of $g(x)$,

$$
\begin{gathered}
g^{(n)}(x) \\
=e^{4 x} \sum_{k=0}^{n}\left(\begin{array}{l}
n \\
k
\end{array}\right) 4^{n-k} \sum_{m=0}^{\infty} \frac{(-1)^{m}\left(-10 m-\frac{5}{3}\right)}{2 m+1} x^{-10 m-\frac{5}{3}-k}
\end{gathered}
$$

Thus, the 12-th order derivative value of $g(x)$ at $x=7$,

$$
\begin{gathered}
g^{(12)}(7) \\
=e^{28} \sum_{k=0}^{12}\left(\begin{array}{c}
12 \\
k
\end{array}\right) 4^{12-k} \sum_{m=0}^{\infty} \frac{(-1)^{m}\left(-10 m-\frac{5}{3}\right)}{2 m+1} 7^{-10 m-\frac{5}{3}-k}(35)
\end{gathered}
$$

Verifying the correctness of (35) as follows:

> evalf((D@@12)(g)(7),18);

$$
5.1378320156153295 \cdot 10^{17}
$$

$>\operatorname{evalf}\left(\exp (28) * \operatorname{sum}\left(\operatorname{sum}\left(12 ! /(\mathrm{k} ! *(12-\mathrm{k}) !) * 4^{\wedge}(12-\mathrm{k}) *(-1)^{\wedge} \mathrm{m}\right.\right.\right.$ *product $(-10 * \mathrm{~m}-5 / 3-\mathrm{p}, \mathrm{p}=0 . .(\mathrm{k}-1)) /(2 * \mathrm{~m}+1) * 7 \wedge(-10 * \mathrm{~m}-5 / 3-$ $\mathrm{k}), \mathrm{m}=0$..infinity), $\mathrm{k}=0 . .12), 18$ );

$$
5.13783201561532949 \cdot 10^{17}
$$

3.2.3. If $x<-1$. By Case (3) of Theorem 2, we obtain any $n$-th order derivative of $g(x)$,

$$
\begin{array}{r}
g^{(n)}(x) \\
=\pi e^{4 x} \sum_{k=0}^{n}\left(\begin{array}{l}
n \\
k
\end{array}\right) 4^{n-k}\left(\frac{10}{3}\right)_{k} x^{\frac{10}{3}-k} \\
+e^{4 x} \sum_{k=0}^{n}\left(\begin{array}{l}
n \\
k
\end{array}\right) 4^{n-k} \sum_{m=0}^{\infty} \frac{(-1)^{m}\left(-10 m-\frac{5}{3}\right)_{k}}{2 m+1} x^{-10 m-\frac{5}{3}-k}
\end{array}
$$

Hence, we can determine the 11-th order derivative value of $g(x)$ at $x=-3$,

$$
g^{(11)}(-3)
$$

$$
\begin{aligned}
& =\pi \cdot e^{-12} \sum_{k=0}^{11}\left(\begin{array}{c}
11 \\
k
\end{array}\right) 4^{11-k}\left(\frac{10}{3}\right)_{k}(-3)^{\frac{10}{3}-k} \\
& +e^{-12} \sum_{k=0}^{11}\left(\begin{array}{c}
11 \\
k
\end{array}\right) 4^{11-k} \sum_{m=0}^{\infty} \frac{(-1)^{m}\left(-10 m-\frac{5}{3}\right)_{k}}{2 m+1}(-3)
\end{aligned}
$$

Using Maple to verify the correctness of (37).

>evalf((D@@11)(g)(-3),18);

\section{$-82.7423991949767509$}

$>\operatorname{evalf}(\mathrm{Pi} * \exp (-12) * \operatorname{sum}(11 ! /(\mathrm{k} ! *(11-\mathrm{k}) !) * 4 \wedge(11-\mathrm{k}) *$ product $\left.(10 / 3-\mathrm{j}, \mathrm{j}=0 . .(\mathrm{k}-1))^{*}\left(3^{\wedge} 10\right)^{\wedge}(1 / 3)^{*}(-1 / 3)^{\wedge} \mathrm{k}, \mathrm{k}=0 . .11\right)+\exp (-12)$ $* \operatorname{sum}\left(\operatorname{sum}\left(11 ! /(\mathrm{k} ! *(11-\mathrm{k}) !) * 4 \wedge(11-\mathrm{k})^{*}(-1)^{\wedge} \mathrm{m}^{*} \operatorname{product}\left(-10^{*}\right.\right.\right.$ $\mathrm{m}-5 / 3-\mathrm{p}, \mathrm{p}=0 . .(\mathrm{k}-1)) /(2 * \mathrm{~m}+1)^{*}\left(1 / 3^{\wedge} 10\right)^{\wedge} \mathrm{m} *\left(-(1 / 243)^{\wedge}(1 / 3)\right)^{*}$ $(-1 / 3)^{\wedge} \mathrm{k}, \mathrm{m}=0$. .infinity), $\left.\left.\mathrm{k}=0 . .11\right), 18\right)$;

\section{$-82.7423991949767511$}

\section{Conclusion}

As mentioned, the Leibniz differential rule, the differentiation term by term, and the integration term by term play significant roles in the theoretical inferences of this study. In fact, the applications of these theorems are extensive, and can be used to easily solve many difficult problems; we endeavor to conduct further studies on related applications. On the other hand, Maple also plays a vital assistive role in problem-solving. In the future, we will extend the research topic to other calculus and engineering mathematics problems and solve these problems by using Maple. These results will be used as teaching materials for Maple on education and research to enhance the connotations of calculus and engineering mathematics.

\section{REFERENCES}

[1] F. Garvan, The Maple Book, London: Chapman \& Hall/CRC, 2001.

[2] C. Tocci and S. G. Adams, Applied Maple for Engineers and Scientists, Boston: Artech House, 1996.

[3] M. L. Abell and J. P. Braselton, Maple by Example, 3rd ed., New York: Elsevier Academic Press, 2005.

[4] R. J. Stroeker and J. F. Kaashoek, Discovering Mathematics with Maple : An Interactive Exploration for Mathematicians, Engineers and Econometricians, Basel: Birkhauser Verlag, 1999.

[5] D. Richards, Advanced Mathematical Methods with Maple, New York: Cambridge University Press, 2002.

[6] J. S. Robertson, Engineering Mathematics with Maple, New York: McGraw-Hill, 1996.

[7] C. T. J. Dodson and E. A. Gonzalez, Experiments in 
Mathematics Using Maple, New York: Springer-Verlag, 1995.

[8] A. Griewank and A. Walther, Evaluating Derivatives: Principles and Techniques of Algorithmic Differentiation, 2nd ed., SIAM, Philadelphia, 2008.

[9] M. Wagner, A. Walther, and B. J. Schaefer, On the efficient computation of high-order derivatives for implicitly defined functions, Computer Physics Communications, Vol. 181, Issue. 4, pp. 756-764, 2010.

[10] M. A. Patterson, M. Weinstein, and A. V. Rao, An efficient overloaded method for computing derivatives of mathematical functions in MATLAB, ACM Transactions on Mathematical Software (TOMS), Vol. 39, Issue. 3, 2013.

[11] C. -H. Yu, A study on some differential problems with Maple, Proceedings of the 6th IEEE/International Conference on Advanced Infocomm Technology, No. 00291, 2013.

[12] C. - H. Yu, Using Maple to evaluate the derivatives of some functions, International Journal of Research in Computer Applications and Robotics, Vol. 1, Issue. 4, pp. 23-31, 2013.

[13] C. -H. Yu, Application of Maple on solving some differential problems, Proceedings of IIE Asian Conference 2013, Vol. 1, pp. 585-592, 2013.

[14] C. - H. Yu, The differential problem of some functions, International Journal of Computer Science and Mobile
Applications, Vol. 1, Issue. 1, pp. 31-38, 2013.

[15] C. -H. Yu, The derivatives of some functions, International Journal of Research in Information Technology, Vol. 1, Issue. 8, pp. 15-23, 2013.

[16] C. -H. Yu, Evaluating the derivatives of trigonometric functions with Maple, International Journal of Research in Computer Applications and Robotics, Vol. 1, Issue. 4, pp. 23-28, 2013.

[17] C. -H. Yu, A Study on the differential problem, International Journal of Research in Aeronautical and Mechanical Engineering, Vol. 1, Issue. 3, pp. 52-57, 2013.

[18] C. -H. Yu, The differential problem of two types of functions, International Journal of Computer Science and Mobile Computing, Vol. 2, Issue. 7, pp. 137-145, 2013.

[19] C. - H. Yu, Evaluating the derivatives of two types of functions, International Journal of Computer Science and Mobile Computing, Vol. 2, Issue. 7, pp. 108-113, 2013.

[20] C. - H. Yu, The differential problem of four types of functions, Journal of Kang-Ning, vol. 15, pp. 51-63, 2013.

[21] C. -H. Yu, A study on the differential problems using Maple, International Journal of Computer Science and Mobile Computing, Vol. 2, Issue. 7, pp. 7-12, 2013.

[22] T. M. Apostol, Mathematical Analysis, 2nd ed., Boston: Addison-Wesley, 1975.. 